Canad. Math. Bull. Vol. 18 (1), 1975

\title{
FUNCTIONAL EQUATIONS OF DIRICHLET SERIES DERIVED FROM NON-ANALYTIC AUTOMORPHIC FORMS OF A CERTAIN TYPE
}

\author{
BY \\ V. VENUGOPAL RAO
}

Let $f(\tau)$ be a complex valued function, defined and analytic in the upper half of the complex $\tau$ plane $(\tau=x+i y, y>0)$, such that $f(\tau+\lambda)=f(\tau)$ where $\lambda$ is a positive real number and $f(-1 / \tau)=\gamma(-i \tau)^{k} f(\tau), k$ being a complex number. The function $(-i \tau)^{k}$ is defined as $\exp (k \log (-i \tau)$ where $\log (-i \tau)$ has the real value when $-i \tau$ is positive. Every such function is said to have signature $(\lambda, k, \gamma)$ in the sense of E. Hecke [1] and has a Fourier expansion of the type $f(\tau)=a_{0}+$ $\sum a_{n} \exp (2 \pi i n / \lambda),(n=1,2, \ldots)$, if we further assume that $f(\tau)=O\left(|y|^{-c}\right)$ as $y$ tends to zero uniformly for all $x, c$ being a positive number. Hecke, then considers the Dirichlet series $\phi(s)=\sum a_{n} n^{-s}(n=1,2, \ldots)$ which has a finite abscissa of absolute convergence and shows [1] that $\phi(s)$ has an analytic continuation in the complex $s$ plane and that it is analytic for all $s$ except for a possible simple pole at $s=k$. Hecke further shows [1] that $\phi(s)$ satisfies the functional equation

$$
(2 \pi / \lambda)^{-s} \Gamma(s) \phi(s)=\gamma(2 \pi / \lambda)^{k-s} \Gamma(k-s) \phi(k-s) .
$$

It is well known that functions with signature $(\lambda, k, \gamma)$ arise from various problems in number theory and in particular from the theory of integral, symmetric, positive definite quadratic forms and their associated theta series. The corresponding theory of integral, indefinite, quadratic forms has been considered by C. L. Siegel [7] and the "theta series" for an integral, indefinite quadratic form turns out to be a "non-analytic" automorphic form, when we exclude certain special types of indefinite quadratic forms. The non-analytic automorphic forms of the type occurring in the work of Siegel [7] have been characterized by H. Maass [2] who proved analogues of the above mentioned results of Hecke [1]. Maass obtains in particular a functional equation for the Dirichlet series formed from the "Fourier coefficients of the non-analytic automorphic form". The "non-analytic automorphic form" depends on two complex parameters $\alpha$ and $\beta$, which we will call the "parameters of the non-analytic automorphic form". (In particular we obtain a classical analytic automorphic form in the sense of Hecke whenever $\beta=0$ ). The functional equation of Maass is a generalization of (1) and involves certain hypergeometric functions (depending on the parameters $\alpha$ and $\beta$ ) instead of the gamma functions occurring in (1). Since the functional equation of a rational indefinite, quadratic form as defined by Siegel [6] involved only gamma functions, it is natural to ask if the hypergeometric functions occurring in the functional equation of Maass can

Received by the editors January 10, 1973 and, in revised form, December 12, 1973. 
be expressed in terms of gamma functions only at least in some special cases, if not in all cases. In fact Maass [2] himself has pointed out three such cases corresponding to $\alpha=0, \beta=0$ and $\alpha=\beta$. In this paper we point out one more case and this we state as a

THEOREM. Let the parameters of the non-analytic automorphic form $\alpha$ and $\beta$ be such that $\alpha-\beta$ is a rational integer. Then the functional equation of the associated Dirichlet series can be expressed in terms of gamma functions.

The statement of the above theorem needs clarification and we next define the non-analytic automorphic form and the associated Dirichlet series.

Let $z$ denote a complex variable, $z=x+i y, x$ and $y$ real and $w=\bar{z}=x-i y$. We consider a pair of complex valued functions $f(z, w)$ and $g(z, w)$ defined in the upper half plane $y>0$ which are solutions of the elliptic partial differential equation

$$
y^{2}\left(\frac{\partial^{2} v}{\partial x^{2}}+\frac{\partial^{2} v}{\partial y^{2}}\right)-(\alpha-\beta) i y \frac{\partial v}{\partial x}+(\alpha+\beta) y \frac{\partial v}{\partial y}=0
$$

$\alpha$ and $\beta$ being complex numbers and having the following properties:

$$
\left\{\begin{array}{l}
f(z+\lambda, w+\lambda)=e^{2 \pi i b_{1}} f(z, w), \\
g(z+\lambda, w+\lambda)=e^{2 \pi i b_{2}} g(z, w),
\end{array}\right.
$$

$\lambda$ being a positive real number and $0 \leq b_{i}<1(i=1,2$,);

$$
g(-1 / z,-1 / w)=\gamma(-i z)^{\alpha}(i w)^{\beta} f(z, w) .
$$

where $\gamma= \pm 1$ and $(-1 z)^{\alpha},(i w)^{\beta}$ are defined by their principal values;

$$
\left\{\begin{array}{l}
f(z, w)=O\left(y^{\lambda_{1}}\right) \text { and } g(z, w)=O\left(y^{\lambda_{2}}\right) \text { as } y \rightarrow \infty \\
f(z, w)=O\left(y^{-\mu_{1}}\right) \text { and } g(z, w)=O\left(y^{-\mu_{2}}\right) \text { as } y \rightarrow \infty
\end{array}\right.
$$

where $\lambda_{i}$ and $\mu_{i}(i=1,2)$ are positive constants and the estimates are uniform in $-\infty<x<\infty$.

It then follows from a result of Maass [2, Hilfassatz 8] that

(6) $f_{1}(x, y) \equiv f(z, w)=a_{0} u(y, \alpha+\beta)+b_{0}$

and

$$
+\sum_{t \neq 0, t=b_{1}(\bmod 1)} a_{t} W\left(\frac{2 \pi|t|}{\lambda} y ; \alpha, \beta, \operatorname{sgn} t\right) e^{2 \pi i t x / \lambda}
$$

(7) $g_{1}(x, y) \equiv g(z, w)=c_{0} u(y, \alpha+\beta)+d_{0}$

$$
+\sum_{t \neq 0, t \equiv b_{2}(\bmod 1)} b_{t} W\left(\frac{2 \pi|t|}{\lambda} y ; \alpha, \beta, \operatorname{sgn} t\right) e^{2 \pi i t x / \lambda}
$$

the series on the right of (6) and (7) being absolutely convergent in the upper half plane $y>0$, where

$$
\begin{gathered}
u(y, \gamma)=\frac{y^{1-\gamma}}{1-\gamma}=\sum_{n=1}^{\infty} \frac{(\log y)^{n}}{n !}(1-\gamma)^{n-1}, \\
W(y ; \alpha, \beta, \varepsilon)=y^{-(\alpha+\beta) / 2} W_{(\alpha-\beta) / 2,(\alpha+\beta-1) / 2}(2 y),(\varepsilon= \pm 1),
\end{gathered}
$$


$W_{1, m}$ being the Whittaker solution of the confluent hypergeometric differential equation in the reduced form [4, p. 296], and sgn $t= \pm 1$ according as $t>0$ or $t<0$ respectively.

We introduce the four Dirichlet series

$$
\begin{array}{ll}
\phi_{1}(s)=\sum_{t>0} \frac{a_{t}}{t^{s}}, & \psi_{1}(s)=\sum_{t>0} \frac{a_{-t}}{t^{s}}, \\
\phi_{2}(s)=\sum_{t>0} \frac{b_{t}}{s}, & \psi_{2}(s)=\sum_{t>0} \frac{b_{-t}}{t^{s}},
\end{array}
$$

where $s$ is a complex variable and $t^{s}=\mathrm{e}^{(s \log t)}$, with $\log t$ real. In view of the estimates (5), the four Dirichlet series in (8) have finite abscissa of absolute convergence. Further it is known that [2] they can be continued analytically into the entire complex $s$ plane and the resulting functions are meromorphic. The functions defined by the Dirichlet series in (8) satisfy the following functional equation.

Let

$$
\begin{gathered}
\Gamma(s ; \alpha, \beta)=\int_{0}^{\infty} W(y ; \alpha, \beta, 1) y^{s-1} d y \\
\xi_{i}(s)=\left(\frac{2 \pi}{\lambda}\right)^{-s}\left\{\Gamma(s ; \alpha, \beta) \phi_{i}(s)+\Gamma(s ; \beta, \alpha) \psi_{i}(s)\right\}
\end{gathered}
$$

and

Then

$$
\begin{aligned}
\eta_{i}(s)= & \left(\frac{2 \pi}{\lambda}\right)^{-(s+1)}\left\{\Gamma(s+1 ; \alpha, \beta)-\frac{1}{2}(\alpha-\beta) \Gamma(s ; \alpha, \beta)\right\} \phi_{i}(s) \\
& -\left(\frac{2 \pi}{\lambda}\right)^{-(s+1)}\left\{\Gamma(s+1 ; \beta, \alpha)+\frac{1}{2}(\alpha-\beta) \Gamma(s ; \beta, \alpha)\right\} \psi_{i}(s), \quad(i=1,2) .
\end{aligned}
$$

$$
\xi_{1}(\alpha+\beta-s)=\gamma \xi_{2}(s) \text { and } \eta_{1}(\alpha+\beta-s)=-\gamma \eta_{2}(s) .
$$

LEMma 1. The function $\phi_{1}(s)$ has the functional equation $2 \Gamma(s) \Gamma(s+1-\alpha-\beta) \phi_{1}(s)$

where

$$
=\gamma(2 \pi / \lambda)^{2 s-\alpha-\beta}\left\{\lambda(\alpha, \beta, s) \phi_{2}(\alpha+\beta-s)+\mu(\alpha, \beta, s) \psi_{2}(\alpha+\beta-s)\right\},
$$

(14) $\lambda(\alpha, \beta, s)=\Gamma(s+1 ; \beta, \alpha) \Gamma(\alpha+\beta-s ; \alpha, \beta)-\Gamma(s ; \beta, \alpha) \Gamma(\alpha+\beta-s+1 ; \alpha, \beta)$,

and

(15) $\mu(\alpha, \beta, s)=\Gamma(s+1 ; \beta, \alpha) \Gamma(\alpha+\beta-s ; \beta, \alpha)+\Gamma(s ; \beta, \alpha) \Gamma(\alpha+\beta-s+1 ; \beta, \alpha)$ $+(\alpha-\beta) \Gamma(s ; \beta, \alpha) \Gamma(\alpha+\beta-s ; \beta, \alpha)$.

Proof. This is the same as lemma 3 of [5]. We remark that lemma 3 of [5] itself is based on a result of Maass [3, p. 300]. 
Lemma 2. With $W(u ; \alpha, \beta, 1)$ as defined in $(8)$ and $W^{(r)}(u ; \alpha, \beta, 1)$ denoting the rth derivative of $W$ with respect to $u$ and $W^{(0)}=W$,

$$
\sum_{r=0}^{n}\left(\begin{array}{l}
n \\
r
\end{array}\right) W^{(n-r)}(u ; \alpha, \beta, 1)=(-1)^{n}(\beta)_{n} 2^{n / 2} W(u ; \alpha, \beta+n, 1),
$$

where $\left(\begin{array}{l}n \\ r\end{array}\right)$ denotes the usual binomial coefficient and

$$
(\beta)_{n}=\beta(\beta+1) \cdots(\beta+n-1) .
$$

Proof. We start with the known formula [4, p. 301]

$$
\frac{d^{n}}{d z^{n}}\left[e^{z / 2} z^{-\mu-1 / 2} W_{\kappa, \mu}(z)\right]=(-1)^{n}\left(\frac{1}{2}+\mu-\kappa\right)_{n} e^{z / 2} z^{-\mu-(n / 2)-1 / 2} W_{\kappa-n / 2, \mu+n / 2}(z)
$$

in which we set $\kappa=\frac{1}{2}(\alpha-\beta), \mu=\frac{1}{2}(\alpha+\beta-1), z=y$ and use the definition (8). We then obtain

$$
\begin{aligned}
\frac{d^{n}}{d y^{n}}\left[e ^ { y / 2 } 2 ^ { - ( \alpha + \beta ) / 2 } W \left(\frac{y}{2}\right.\right. & ; \alpha, \beta, 1)] \\
& =(-1)^{n}(\beta)_{n} e^{y / 2} y^{-(n / 2)-(\alpha+\beta) / 2} W_{(\alpha-\beta) / 2-n / 2,(\alpha+\beta-1) / 2+n / 2}(2 y)
\end{aligned}
$$

The left side of (16) is

$$
2^{-(\alpha+\beta) / 2} \sum_{r=0}^{n}\left(\begin{array}{l}
n \\
r
\end{array}\right) 2^{-r} e^{y / 2} W^{(n-r)}\left(\frac{y}{2} ; \alpha, \beta, 1\right) 2^{-(n-r)},
$$

and the right side of (16), by the use of (8) is

$$
(-1)^{n}(\beta)_{n} e^{y / 2} 2^{-(n / 2)-(\alpha+\beta) / 2} W\left(\frac{y}{2} ; \alpha, \beta+n, 1\right) .
$$

Writing $y=2 u$, we get lemma 2 .

LEMMA 3.

and

$$
W^{(r)}(u ; \alpha, \beta, 1)=O\left(y^{-\operatorname{Re}(\beta)} e^{-y}\right), \text { for } y \rightarrow \infty(y \text { real }),
$$

with

$$
W^{(r)}(u ; \alpha, \beta, 1)=O\left(y^{-K_{r}}\right), \quad \text { for } \quad y \rightarrow 0(y \text { real }),
$$

$$
K_{r}>\operatorname{Re} \frac{1}{2}(\alpha+\beta+r)+\operatorname{Re}\left|\frac{1}{2}(\alpha+\beta+r)\right|-1 .
$$

Proof. It is known [2, p. 247] that

$$
W(y ; \alpha, \beta, \varepsilon) \sim 2^{(\alpha-\beta) \varepsilon / 2} y^{-\{(\alpha+\beta)+(\beta-\alpha) \varepsilon\} / 2} e^{-y},
$$

as $y \rightarrow \infty$. Further, from the power series expansion of the Whittaker function, it follows that

$$
W(y ; \alpha, \beta, 1)=O\left(y^{-K}\right), \text { for real } y \rightarrow 0,
$$

with $K>\operatorname{Re} \frac{1}{2}(\alpha+\beta)+\operatorname{Re}\left|\frac{1}{2}(\alpha+\beta)\right|-1$. Lemma 3 then follows by using lemma 2 and the induction argument. 
Lemma 4. Let $\Gamma(s ; \alpha, \beta)$ be as defined in (10) and $k$, a non-negative integer. Then

$$
\Gamma(s ; \beta+k, \beta)=2^{-\beta-(k / 2)} \Gamma(s) \sum_{r=0}^{k}\left(\begin{array}{l}
k \\
r
\end{array}\right) \frac{\Gamma\left(\frac{s-r+1}{2}-\beta\right)}{\Gamma\left(\frac{s-r+1}{2}\right)}
$$

and

$$
\Gamma(s ; \beta, \beta+k)=2^{-\beta-(k / 2)} \frac{\Gamma(\beta)}{\Gamma(\beta+k)} \Gamma(s) \sum_{r=0}^{k}(-1)^{r}\left(\begin{array}{l}
k \\
r
\end{array}\right) \frac{\Gamma\left(\frac{s-r+1}{2}-\beta\right)}{\Gamma\left(\frac{s-r+1}{2}\right)} .
$$

Proof. If $k \geq 0$ is an integer, it is known [2, p. 251] that

$$
W(y ; \beta+k, \beta, \varepsilon)
$$

$$
=(-1)^{k} 2^{(1-k) / 2} \pi^{-1 / 2} \frac{\Gamma(\beta)}{\Gamma\left(\beta+\frac{k(1-\varepsilon)}{2}\right)} e^{\varepsilon y} \frac{d^{k}}{d y^{k}}\left(e^{-\varepsilon y} y^{1 / 2-\beta} K_{\beta-1 / 2}(y)\right),
$$

where $K_{\mu}(x)$ denotes the usual Bessel function of imaginary argument. Choosing $k=0$ in (19), it follows that

$$
W(y ; \beta, \beta, \varepsilon)=\left(\frac{2}{\pi}\right)^{1 / 2} y^{1 / 2-\beta} K_{\beta-1 / 2}(y) .
$$

From (19) and (20), we obtain

(21) $W(y ; \beta+k, \beta, \varepsilon)=(-1)^{k} 2^{-k / 2} \frac{\Gamma(\beta)}{\Gamma\left(\beta+\frac{k(1-\varepsilon)}{2}\right)} e^{\varepsilon y} \frac{d^{k}}{d y^{k}}\left(e^{-\varepsilon y} W(y ; \beta, \beta, \varepsilon)\right)$.

We have

$$
\Gamma(s ; \beta+k, \beta)=\int_{0}^{\infty} y^{s-1} W(y ; \beta+k, \beta, 1) d y,
$$

where the integral on the right of (22) converges for $\operatorname{Re} s>K_{0}$, with $K_{0}$ as in lemma 3. We first consider the case $k>0$. It follows from (21) that the integral on the right of (22) is

$$
\begin{aligned}
(-1)^{k} 2^{-k / 2} \int_{0}^{\infty} y^{s-1} e^{y} \frac{d^{k}}{d y^{k}}\left(e^{-y} W(y ; \beta, \beta, 1)\right) d y & \\
= & (-1)^{k} 2^{-k / 2} \int_{0}^{\infty} y^{s-1}\left\{\sum_{r=0}^{k}(-1)^{r}\left(\begin{array}{l}
k \\
r
\end{array}\right) W^{(k-r)}(y ; \beta, \beta, 1)\right\} d y .
\end{aligned}
$$


Now

$$
\begin{aligned}
\int_{0}^{\infty} y^{s-1} W^{(k-r)}(y ; & \beta, \beta, 1) d y \\
= & {\left[y^{s-1} W^{(k-r-1)}(y ; \beta, \beta, 1)\right]_{0}^{\infty}-(s-1) \int_{0}^{\infty} y^{s-2} W^{(k-r-1)}(y ; \beta, \beta, 1) d y } \\
& =-(s-1) \int_{0}^{\infty} y^{s-2} W^{(k-r-1)}(y ; \beta, \beta, 1) d y,
\end{aligned}
$$

in the half plane $\operatorname{Re} s>1+K_{k-r-1}$, in view of lemma 3

$$
=(-1)^{k-r}(s-1)(s-2) \cdots(s-k+r) \int_{0}^{\infty} y^{s-k+r-1} W^{(0)}(y ; \beta, \beta, 1),
$$

by induction

$$
=(-1)^{k-r}(s-1)(s-2) \cdots(s-k+r) \Gamma(s-k+r ; \beta, \beta) .
$$

We therefore have

$$
\begin{aligned}
2^{k / 2} \Gamma(s ; \beta+k, \beta) & =\sum_{r=0}^{k}\left(\begin{array}{l}
k \\
r
\end{array}\right)(s-1) \cdots(s-k+r) \Gamma(s-k+r ; \beta, \beta) \\
& =\sum_{r=0}^{k}\left(\begin{array}{l}
k \\
r
\end{array}\right)(s-1) \cdots(s-r) \Gamma(s-r ; \beta, \beta) \\
& =\sum_{r=0}^{k}\left(\begin{array}{l}
k \\
r
\end{array}\right) \frac{\Gamma(s)}{\Gamma(s-r)} \Gamma(s-r ; \beta, \beta) .
\end{aligned}
$$

Since

and

it follows that

$$
\Gamma(s ; \alpha, \alpha)=\pi^{-1 / 2} 2^{s-\alpha-1} \Gamma\left(\frac{s}{2}\right) \Gamma\left(\frac{s+1}{2}-\alpha\right),
$$

$$
\Gamma(s)=\pi^{-1 / 2} 2^{s-1} \Gamma\left(\frac{s}{2}\right) \Gamma\left(\frac{s+1}{2}\right)
$$

and obtain

$$
\frac{\Gamma(s-r ; \beta, \beta)}{\Gamma(s-r)}=2^{-\beta} \frac{\Gamma\left(\frac{s-r+1}{2}-\beta\right)}{\Gamma\left(\frac{s-r}{2}\right)} .
$$

Inserting (25) in (23), we obtain (17), valid in some half plane. By analytic continuation (17) remains valid in the domain of regularity of $\Gamma(s ; \beta+k, \beta)$.

For the proof of (18) we observe that

$$
W(y ; \alpha, \beta, 1)=W(y, \beta, \alpha,-1),
$$


We then use (19) with $\varepsilon=-1$ and deduce for the right side of (25)

$$
\begin{aligned}
(-1)^{k} 2^{-k / 2} \frac{\Gamma(\beta)}{\Gamma(\beta+k)} \int_{0}^{\infty} y^{s-1} e^{-y} \frac{d^{k}}{d y^{k}}\left(e^{y} W(y ; \beta, \beta, 1) d y\right. \\
=(-1)^{k} 2^{-k / 2} \frac{\Gamma(\beta)}{\Gamma(\beta+k)} \sum_{r=0}^{k}\left(\begin{array}{l}
k \\
r
\end{array}\right) \int_{0}^{\infty} y^{s-1} W^{(k-r)}(y ; \beta, \beta, 1) d y \\
=(-1)^{k} 2^{-k / 2} \frac{\Gamma(\beta)}{\Gamma(\beta+k)} \sum_{r=0}^{k}\left(\begin{array}{l}
k \\
r
\end{array}\right)(-1)^{k-r}(s-1) \cdots(s-k+r) \Gamma(s-k+r ; \beta, \beta) \\
=2^{-k / 2} \frac{\Gamma(\beta)}{\Gamma(\beta+k)} \sum_{r=0}^{k}\left(\begin{array}{l}
k \\
r
\end{array}\right)(-1)^{r} \frac{\Gamma(s)}{\Gamma(s-r)} \Gamma(s-r ; \beta, \beta) .
\end{aligned}
$$

We then obtain (18) by a procedure similar to the proof of (17). So far $k>0$. If $k=0$, the lemma follows from (24).

THEOREM. If $\beta=\alpha \pm k$, where $k \geq 0$ is an integer, the functional equation of $\phi_{1}(s)$ can be expressed in terms of gamma functions.

Proof. In view of lemma 4 , the functions $\lambda(\alpha, \beta, s)$ and $\mu(\alpha, \beta, s)$, defined by (14) and (15) can be expressed in terms of gamma functions whenever $\beta=\alpha \pm k$, and $k>0$. If $k=0$, we derive the same conclusion by using (25). Hence by lemma 1 , the functional equation of $\phi_{1}(s)$ can be expressed in terms of gamma functions.

\section{REMARKS.}

1. The expressions (17) and (18) for $\Gamma(s ; \alpha, \beta)$ and $\Gamma(s ; \beta, \alpha)$ respectively in the case $\beta=\alpha+k$ strongly suggest that they can be further simplified when $\beta$ is an integer. This fact has already been confirmed by Satz 6 of Maass [2].

2. The functional equations (13) yield functional equations for $\phi_{1}(s), \psi_{1}(s)$, $\phi_{2}(s)$ and $\psi_{2}(s)$. Lemma 1 relates $\phi_{1}(s)$ with $\phi_{2}(\alpha+\beta-s)$ and $\psi_{2}(\alpha+\beta-s)$. There exists an analogous relation between $\psi_{1}(s)$ and $\phi_{2}(\alpha+\beta-s), \psi_{2}(\alpha+\beta-s)$. This can be derived in a manner similar to the functional equation of $\phi_{1}(s)$ as stated by lemma 1, which turns out to be

$$
2 \Gamma(s) \Gamma(s+1-\alpha-\beta) \psi_{1}(s)=A(\alpha, \beta, s) \phi_{2}(\alpha+\beta-s)+B(\alpha, \beta, s) \psi_{2}(\alpha+\beta-s),
$$

where

$$
A(\alpha, \beta, s)=\Gamma(\alpha+\beta-s ; \alpha, \beta) \Gamma(s+1 ; \alpha, \beta)+\Gamma(s ; \alpha, \beta) \Gamma(\alpha+\beta-s+1 ; \alpha, \beta) .
$$

and

$$
\begin{array}{r}
B(\alpha, \beta, s)=\Gamma(\alpha+\beta-s ; \beta, \alpha) \Gamma(s+1 ; \alpha, \beta)-\Gamma(s ; \alpha, \beta) \Gamma(\alpha+\beta-s+1 ; \beta, \alpha) \\
+(\beta-\alpha) \Gamma(s ; \alpha, \beta) \Gamma(\alpha+\beta-s ; \alpha, \beta) .
\end{array}
$$

In view of lemma $4, A(\alpha, \beta, s)$ and $B(\alpha, \beta, s)$ can be expressed once again in terms of gamma functions when $\beta=\alpha \pm k$, where $k \geq 0$ is an integer. 


\section{BIBLIOGRAPHY}

1. E. Hecke, Über die Bestimmung Dirichletscher Reihen durch ihre Funchonalgleichung, Math. Annalen 112 (1936), 664-699.

2. H. Maass, Die Differentialgleichungen in der Theorie der elliptischen Modulfunktionen, Math. Annalen, 125 (1953), 235-263.

3. H. Maass, Über die räumliche Verteilung der Punkte in Gittern mit indefiniter Metrik, Math. Annalen, 138 (1959), 287-315.

4. W. Magnus, F. Oberhettinger, and R. P. Soni, Formulas and Theorems for the Special Functions of Mathematical Physics, Springer-Verlag, New York, 1966.

5. V. V. Rao, Averages involving Fourier Coefficients of Non-Analytic Automorphic Forms, Canadian Mathematical Bulletin, 13(2) (1970), 187-198.

6. C. L. Siegel, Über die Zetafunktionen indefiniter quadratischer Formen II, Math. Zeit, 44 (1939), 398-426.

7. C. L. Siegel, Indefinite Quadratischer Formen and Funktionentheorie I, Math. Annalen, 124 (1951), 17-54.

University of Saskatchewan, Regina Campus, Regina, Saskatchewan, CaNada 